\title{
FÖRSTER TRANSFER CALCULATIONS BASED ON CRYSTAL STRUCTURE DATA FROM Agmenellum quadruplicatum C-PHYCOCYANIN
}

\author{
Kenneth Sauer ${ }^{1}$, Hugo Scheer ${ }^{2, *}$ and Peter Sauer ${ }^{3}$ \\ 'Chemistry Department and Chemical Biodynamics Laboratory, Lawrence Berkeley Laboratory, \\ University of California, Berkeley, CA 94720 , USA, ${ }^{2}$ Botanisches Institut der Universität \\ München, Menzinger Straße 67, 8000 München 19, West Germany and ${ }^{3}$ Carleton College, \\ Northfield, MN 50557, USA
}

(Received 21 November 1986; accepted 5 March 1987)

\begin{abstract}
Excitation energy transfer in C-phycocyanin is modeled using the Förster inductive resonance mechanism. Detailed calculations are carried out using coordinates and orientations of the chromophores derived from X-ray crystallographic studies of C-phycocyanin from two different species (Schirmer et al., J. Mol. Biol. 184, 257-277 (1985) and ibid., 188, 651-677 (1986)). Spectral overlap integrals are estimated from absorption and fluorescence spectra of C-phycocyanin of Mastigocladus laminosus and its separated subunits. Calculations are carried out for the $\beta$-subunit, $\alpha \beta$-monomer, $(\alpha \beta)_{3}$-trimer and $(\alpha \beta)_{6}$-hexamer species with the following chromophore assignments: $\beta 155=$ ' $s$ ' (sensitizer), $\beta 84=$ ' $\mathrm{f}$ ' (fluorescer) and $\alpha 84=$ ' $\mathrm{m}$ ' (intermediate) $\ddagger$. The calculations show that excitation transfer relaxation occurs to $\geqslant 98 \%$ within $200 \mathrm{ps}$ in nearly every case; however, the rates increase as much as 10 -fold for the higher aggregates. Comparison with experimental data on fluorescence decay and depolarization kinetics from the literature shows qualitative agreement with these calculations. We conclude that Förster transfer is sufficient to account for all of the observed fluorescence properties of C-phycocyanin in aggregation states up to the hexamer and in the absence of linker polypeptides.
\end{abstract}

\section{INTRODUCTION}

The light harvesting pigment proteins associated with photosynthesis function by absorbing light and transferring the resulting electronic excitation energy to one or more photosynthetic reaction centers. This energy transfer process, which is typically complete within about $10^{-16} \mathrm{~s}$, has been described as involving a sequence of exciton delocalization among strongly interacting pigment chromophores followed by Förster inductive resonance transfer between pigment-protein complexes (Sauer, 1986). This two-stage mechanism is particularly appropriate in the case of chlorophyll-containing lightharvesting complexes, where spectroscopic band splittings and conservative sigmoidal $\mathrm{CD} \dagger$ spectra are thought to reflect the exciton coupling interactions (Sauer, 1975; Cogdell and Scheer, 1985). Close proximity $(\leqslant 2.0 \mathrm{~nm})$ of the chromophores is thought to be essential for such exciton coupling to play a significant role in the excitation delocalization.

In other antenna types like the phycobiliproteins Förster type transfer is believed to be the dominant mechanism (Scheer, 1981, 1982). The recent publication of molecule coordinates of two C-phycocyanin molecules based on X-ray crystallographic

*To whom correspondence should be addressed.

$\dagger$ Abbreviations: cd, circular dichroism: PC, C-phycocyanin.

$¥$ The corrected amino-acid numbering has been used throughout (Schirmer et al., 1985). determinations (Schirmer et al., 1985; Schirmer et al., 1986) enables detailed excitation transfer calculations to be carried out for the first time on any photosynthetic pigment protein. Because the nearest interchromophore separations (center-to-center) are $2.4 \mathrm{~nm}$ in these molecules and because unambiguous evidence of excitonic coupling is absent in the molecular spectra of isolated, linker-free phycocyanins, we have assumed that excitation transfer in C-phycocyanin occurs solely by the Förster inductive resonance transfer mechanism (Förster, 1948, 1967; Dale and Eisinger, 1975). To calculate transfer rates using the Förster formalism, we have used interchromophore distances and chromophore orientations from the crystal structure determination of the hexameric C-phycocyanin from Agmenellum quadruplicatum (Schirmer et al., 1986) which contains 18 phycocyanobilin chromophores per hexameric unit. The trimeric C-phycocyanin from Mastigocladus laminosus (Schirmer et al., 1985 ) is essentially half of this hexameric structure. The spectral overlap integrals were calculated based on an analysis of the absorption and emission spectra of C-phycocyanin and of its $\alpha$ - and $\beta$-subunits. Fluorescence lifetimes were taken from previously published experimental values (Switalski and Sauer, 1984; Hefferle et al., 1984a,b). The model for the excitation transfer calculation and the method of solving the kinetic expressions describing the excited state behavior are reported in detail. Emphasis is given to results which can be compared directly with experimental measurements, such as fluorescence depolarization, photodichroism of ground state 
bleaching and recovery, and wavelength resolved fluorescence decay kinetics. The data are calculated in a fashion that enables them to be compared with measurements made under a variety of experimental conditions.

\section{MATERIALS AND METHODS}

Chromophore spectra. The absorption and fluorescence spectra of the $\alpha 84$ chromophore were obtained from the isolated $\alpha$-subunit. The absorption spectra of the two $\beta$ subunit chromophores were obtained by a deconvolution procedure, which assumed for both $\beta$-chromophores the shape and bandwidth of the $\alpha$-chromophore. The rationale for this approximation was firstly the rather similar, asymmetric shape of bile pigment spectra irrespective of the chromophore conformation and state of protonation. Secondly, it is based on the unusual circular dichroism (CD) spectrum of the $\beta$-subunit, which is similar to that of the $\alpha$-subunit with two exceptions: Its extremum is strongly blue-shifted as compared to the absorption maximum, and the anisotropy is only approx. Forty-five per cent of that of the $\alpha$-subunit. This can be rationalized by the presence of two different $(\beta 1, \beta 2)$ chromophores. One chromophore is strongly optically active (similar to that of the $\alpha$-subunit); this is the one absorbing at shorter wavelengths ( $\beta 2)$ and its absorption maximum should then be close to the CD extremum. The other, optically less active $(<10 \%$ of the $\alpha$-chromophore) chromophore absorbs at longer wavelength $(\beta 1)$. Thirdly, it was observed that the shape of the fluorescence emission curve of the $\beta$-subunit is similar to that of the $\alpha$-subunit. It was originally assumed that this fluorescence was due only to the fluorescing chromophore of the $\beta$-subunit. Our calculations indicate. however, that there is an equilibrium between the two chromophores, with $80-85 \%$ of the excitation residing on the longer wavelength chromophore and the remaining $15-20 \%$ residing on the shorter wavelength one (' $\mathrm{f}$ ' and ' $s$ ' in the Teale and Dale (1970) notation, respectively, see results). The fluorescence of the short wavelength chromophore, is probably the origin of the increase in fluorescence polarisation measured on the short-wavelength side of the emission spectrum of $\beta$-subunits reported earlier by Mimuro et al. (1986). We assumed that its Stokes shift and shape is the same for each chromophore type.

The assignment of the $\beta 84$ and $\beta 155$ chromophores to the two absorption/emission components $(\beta 1, \beta 2)$ of the $\beta$ subunit is not possible a priori. Conflicting spectroscopic results have been discussed by John et al. (1985). Mimuro et al. (1986) have suggested that $\beta 84$ is the chromophore absorbing at longer wavelength, and this assignment has been verified by titration of PC with cysteine-modifying reagents (Siebzehnrübl et al., 1987). The resulting spectral characteristics of the chromophores $\beta 84$ and $\beta 155$, as well as these of $\alpha 84$, are assembled in Table 1 and Fig. 1A. Calculations have been carried out for both possible assignments of the $\beta$-chromophores, with basically similar results. In view of the aforementioned biochemical assignment, only the data corresponding to $\beta 155=\mathrm{s}$ (sensitizer) and $\beta 84=f$ (fluorescer) are published here. We refer to this as the mfs model for the assignment of the $\alpha 84, \beta 84$ and $\beta 155$ chromophores, respectively (see note added in proof).

Calculations. The equations describing the kinetic behavior of the excited electronic state of C-phycocyanin are solved using numerical methods involving an iterative procedure known as the backward Euler method (Shampine and Gordon, 1975). In brief, a model was assumed in which excitation started at time zero on one chromophore in a cluster of $\mathrm{N}$ chromophores within a C-PC molecule, where $N$ was 2 for the $\beta$-subunit, 3 for an $(\alpha \beta)$ monomer, 9 for an $(\alpha \beta)_{3}$-trimer and 18 for an $(\alpha \beta)_{6}$ hexamer. The chromophores within each cluster were assumed to be coupled pairwise by only the Förster inductive resonance interaction. Rate constants $k_{\mathrm{D}} \rightarrow_{\mathrm{A}}$ for excitation transfer between coupled donor, D, and acceptor, A, chromophores were calculated using the usual Förster equation. (The corrected version (Förster, 1967) containing the factor of $\Pi^{5}$ was used in place of $\Pi^{6}$ used originally (Förster, 1948).)

$$
k_{\mathrm{D} \rightarrow \mathrm{A}}=\frac{9 \ln 10 \kappa^{2} \Phi_{\mathrm{D}}}{128 \Pi^{5} \mathrm{~N} n^{4} \tau_{\mathrm{D}} \mathrm{R}_{\mathrm{DA}}^{6}} \int_{0}^{\infty} f_{\mathrm{D}}(v) \epsilon_{\mathrm{A}}(v) v^{-4} \mathrm{~d} v
$$

where $\kappa$ is the orientation factor, $\kappa_{\mathrm{ij}}=\mu_{\mathrm{i}} \mu_{\mathrm{j}}-3\left(\mu_{1} R_{\mathrm{ij}}\right)$ $\left(\mu_{1} R_{\mathrm{ij}}\right)$, with $\mu_{1}$ and $\mu_{1}$ as the transition dipoles of the

Table 1. Spectroscopic properties calculated for chromophores of C-phycocyanin

\begin{tabular}{lcrcc}
\hline & $\begin{array}{c}\lambda_{\text {Amax }} \\
(\mathrm{nm})\end{array}$ & $\begin{array}{c}\epsilon_{\max } \\
\left(\mathrm{cm}^{2} \mathrm{~mol}^{-1}\right)\end{array}$ & $\begin{array}{c}\lambda_{\text {Fmax }} \\
(\mathrm{nm})\end{array}$ & $\Phi_{\mathbf{r}}$ \\
\hline$\alpha 84$ & 617 & $122.0 \times 10^{6}$ & 641 & 0.72 \\
$\beta 84$ & 622 & $73.2 \times 10^{\circ}$ & 644 & 0.48 \\
$\beta 155$ & 598 & $109.0 \times 10^{\circ}$ & 623 & 0.72 \\
\hline
\end{tabular}

(A): Wavelength of maximum absorbance, $\lambda_{\text {Amax }}$ (from Siebzehnrübl et al., 1986), molar absorptivity at maximum absorbance, $\epsilon^{\text {max }}$; wavelength of maximum fluorescence, $\lambda_{\mathrm{F}}{ }^{\text {max }}$; absolute quantum yield of fluorescence, $\Phi^{F}$

(B): Overlap integrals $\left(\times 10^{+10}, \mathrm{~cm}^{6} \mathrm{~mol}^{-1}\right)$ and variations upon a shift of the emission band of the donor by $\pm 2 \mathrm{~nm}$.

\begin{tabular}{lrcc}
\hline \multicolumn{1}{c}{ Acceptor } & \multicolumn{1}{c}{$\alpha 84$} & $\beta 84$ & $\beta 155$ \\
\hline Donor & $7.03(+0.53 /-0.48)$ & $5.36(+0.37 /-0.38)$ & $2.38(+0.31 /-0.36)$ \\
$\alpha 84$ & $6.79(+0.60 /-0.54)$ & $5.16(+0.37 /-0.36)$ & $2.36(+0.30 /-0.27)$ \\
$\beta 84$ & $10.90(+0.30 /-0.40)$ & $7.11(+0.14 /-0.17)$ & $5.53(+0.50 /-0.48)$ \\
$\beta 155$ & & & \\
\hline
\end{tabular}


A

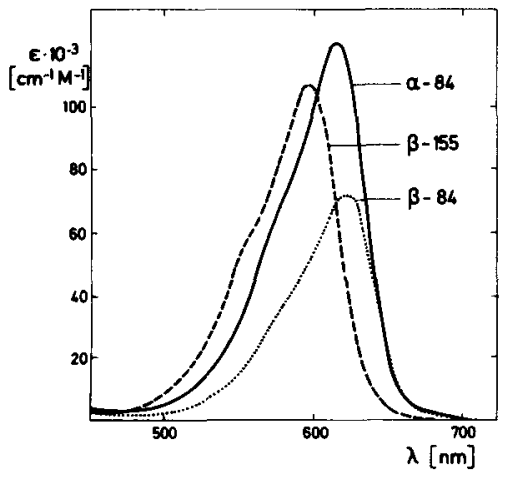

B

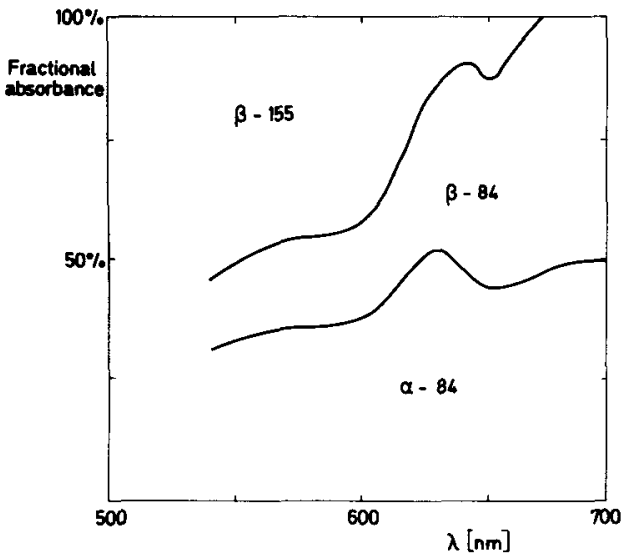

Figure 1. (A) Absorption spectra of the individual chromophores used in the calculations for the overlap integrals. The spectrum of the $\alpha 84$-chromophore has been determined experimentally on the isolated $\alpha$-subunit of PC from Mastigocladus laminosus. The spectra of the $\beta 84$ and $\beta 155$-chromophores are derived from a curve resolution of the absorption spectrum of the isolated $\beta$-subunit of the same organism. The individual components were assumed to have the same shape and bandwidth as the $\alpha 84$ absorption (see Methods for details). (B) Excitation ratios for the three chromophores at different wavelengths across the the long-wavelength absorption band of PC from $M$. laminosus. The values are based on the spectra of the individual chromophores shown in (A).

chromophores $\mathrm{i}$ and $\mathrm{j}$, respectively, and $R_{\mathrm{ij}}$ as the vector connecting them. $\tau_{D}$ is the actual mean lifetime of the excited state and $\Phi_{D}$ is the quantum yield of fluorescence, $\mathrm{n}$ is the refractive index of the medium, $\mathrm{N}$ is Avogadro's number per mole, $R_{\mathrm{DA}}$ is the center-to-center separation of the chromophores (in $\mathrm{cm}$ ), $f_{\mathrm{D}}$ is the spectral distribution of fluorescence of the donor (in quanta, and normalized to unity), $\epsilon_{A}$ is the molar extinction coefficient of the acceptor (in $\mathrm{cm}^{2} \mathrm{~mol}^{-1}$ ) and $v$ is the frequency (in $\mathrm{cm}^{-1}$ ). A refractive index of $n=1.54$ was used to reflect the protein nature of the intervening matrix.

A value of $\tau_{\mathrm{F}}=1.5 \mathrm{~ns}$ has been used for all chromophores; this is an average of the longest lifetimes observed experimentally for $\mathrm{PC}$ in various states of aggregation (Hefferle et al., 1984a,b; Holzwarth, 1986; Switalski and Sauer, 1984). The values for $\kappa$ and for $R_{\mathrm{DA}}$ are taken from Schirmer et al. (1986) without further corrections. The orientation of each chromophore is determined by these authors from the mass distribution of the conjugated atoms; the distances are between the centers of mass (= approximately at atom $\mathrm{C}-10$ ) of these conjugated parts. The calculation is greatly simplified because of the inherent $\mathrm{C}_{3}$ symmetry of C-PC aggregates. There are only three distinct chromophore clases, designated $\alpha 84, \beta 84$ and $\beta 155$. In each of the aggregates that we studied, every chromophore belongs to one of these three classes.

The calculations were carried out in the following fashion: For a cluster of $N$ coupled chromophores, one was chosen to be the location of all of the initial excitation at time zero. Using the Förster relation (Eq. 1) the rate constants were calculated for excitation transfer from the initially excited chromophore to each of the other $N-1$ chromophores in the cluster, as well as for pairwise transfer between all of the other chromophores. A set of $N$ first-order differential equations of first degree was set up to describe the growth and/or decay of excited state population at each chromophore in the cluster, assuming first-order kinetic behavior. In addition, each equation included a single first-order term describing loss of excitation by irreversible processes, such as fluorescence, radiationless internal conversion to the ground state and intersystem crossing to the triplet manifold. Unless otherwise stated, the rate constant governing all these processes taken together was $k_{\mathrm{F}}=0.67 \mathrm{~ns}^{-1}$, corresponding to an average of the observed fluorescence lifetime of approx. $1.5 \mathrm{~ns}$ (vide supra). (By setting this parameter to zero, the calculation then describes the relative redistribution of excitation among the interacting chromophores in the absence of excited state decay.)

A computer program was written and compiled in BASIC to solve by an iterative method for the population of excitation on each of the chromophores in the cluster as a function of time. Although direct solutions of the system of differential equations are possible and less time consuming, we chose an iterative method using up to 10 min computing time (on an IBM-AT) because this allows an easy extraction of data simulating a variety of experimental conditions including polarized spectra. The method used, known as the backward Euler method or the Predictor-Corrector procedure (PECE Method) with $k=1$ in the Adams formulas (Shampine and Gordon, 1975) is sensitive to the time intervals used and to the number of iterations. If either of these is insufficient, then the solutions become unstable at later times and large oscillations begin to occur in the populations. Fortunately, this happens quite suddenly using the backward Euler method, and it is easy to spot. This is less true for the normal Euler method. The step size and number of steps need to be most dense at the beginning, depending considerably on the value of $N$ for the problem being solved. The most demanding calculation that was carried out was for $N=18$; in this case initial step sizes of 0.01 ps were necessary and up to 3500 iterations were included for the interval to 4 ns.

For each value of $N$ and set of model parameters, it was necessary to make three separate calculations. In each case the initial excitation was placed on a chromophore from a different class: $\alpha 84, \beta 84$ or $\beta 155$. Only three such initial conditions were needed, even when $N=9$ or $N=18$, because each of the other possibilities is related to one of the three by symmetry. Once the three calculations were completed, they could be combined to analyze different conditions or to simulate a specific experiment. Because the absorption (excitation) and emission spectra of the three chromophore types are not identical, the extent to which each is initially excited and its fluorescence is detected, depends on the excitation and emission wavelengths, respectively. In an experiment that monitors the isotropic part of fluorescence decay, emission coming from each of the N/3 chromophores of a particular class is equally likely to be detected. Hence. it is relevant to add 
the contributions of all members of the class. When the anisotropic part (parallel polarized fluorescence) is monitored, then the probability of detection depends on the orientations of the emitting chromophores relative to those excited. Measurements on single crystals in a defined orientation produce analogous restrictions.

All such experimental conditions can be simulated in the calculations by the introduction of appropriate factors derived from: (1) The relative excitation probabilities of the different chromophore types at a given wavelength, (2) the relative emission probabilities, and (3) the depolarization of emission due to energy transfer or sample orientation. The former two matrices have been tabulated every $5 \mathrm{~nm}$ from the spectral data of individual chromophores shown in Fig. 1, the last one from the geometrical data of Schirmer et al. (1986). We will explore only the simplest of these applications in this paper. For the calculations shown in Fig. 6, only the first factor has been applied. The matrices for all three factors enter the fluorescence polarization spectra shown in Fig. 7 . The polarization was then averaged and weighted over the calculated decay interval. For the remaining time, a constant polarization was assumed. This assumption seemed to be justified, because equilibration is complete within $\leq 0.5 \mathrm{~ns}$, and the calculations extended to $\geq 5 \mathrm{~ns}$.

\section{RESULTS}

\section{Effect of cluster size}

The consequences of increasing the number of interacting chromophores in a cluster of C-PC can be seen most readily in terms of the rate of loss of excitation from the initially excited chromophore, extrapolated to time zero $\left(k_{0}\right)$. This value is the sum of the first-order rate constants for excitation transfer away from the initially excited chromophore to each of the other $N-1$ chromophores in the cluster, plus that for loss of excitation, $k_{\mathrm{F}}$. For chromophore 1 , this leads to

$$
\left(k_{0}{ }^{1}\right)=k_{12}+k_{13}+\ldots+k_{1 N}+k_{\mathrm{F}}
$$

In Table 2 we compare results for the $\beta$-subunit $(N=2), \alpha \beta$-monomer $(N=3),(\alpha \beta)_{3}$-trimer $(N=9)$ and $(\alpha \beta)_{6}$-hexamer $(N=18)$. The initial slope of the excited state population of chromophore $1 \alpha 84$ chromophore in Figs $5 \mathrm{~B}$ and $\mathrm{C}$ also corresponds to this value. It is clear immediately that excitation relaxes from the initially excited chromophore much more rapidly in the larger cluster than in the smaller ones. This is a direct consequence of the detailed structure of C-PC, and major roles are played by (1) the interchromophore separation, which ranges from 2.36 to $9.26 \mathrm{~nm}$ for the $(\alpha \beta)_{6}$-hexamer and which enters as the sixth power in the Förster expression, (2) the orientation factor, which ranges from 0.12 to 1.85 and enters as square in the Förster expression, and (3) the overlap integrals, which range over a factor of 4.6. The consequences are that the initial loss of excitation is from 9- to over 100-fold faster in the hexamer than in the monomer or the $\beta$-subunit. In the latter clusters the shortest distance of separation is $3.59 \mathrm{~nm}$, between the $\beta 84$ and the $\beta 155$ chromophores; the orientation factor, $\kappa=0.81$, is relatively favorable. In the $\alpha \beta$-monomer the distance of the $\alpha 84$ chromophore from the $\beta 84$ and $\beta 15$ chromophores is 5.75 and $5.46 \mathrm{~nm}$, respectively, and the orientation factor of $\kappa=$ -0.13 for the $\alpha 84 / \beta 84$ interaction is particularly unfavorable. In the $(\alpha \beta)_{3}$-trimer there are significantly closer contacts between $1 \alpha 84$ and $2 \beta 84$ of $2.44 \mathrm{~nm}$, and between $1 \beta 84$ and $2 \beta 84$ or $3 \beta 84$ of $3.57 \mathrm{~nm}$ with a very favorable $\kappa=1.46$. The separation of $1 \alpha 84$ from $2 \beta 155$ is also relatively short at $3.58 \mathrm{~nm}$. The most favorable interactions of all occur in the $(\alpha \beta)_{6}$-hexamer between chromophores in the upper and lower trimers, in this face-to-face structure. The distance between $1 \alpha 84$ and $4 \alpha 84$ is only $2.36 \mathrm{~nm}$ with $\kappa=0.56$; that between $1 \beta 155$ and $6 \beta 155$ is $2.86 \mathrm{~nm}$ with $\kappa=1.15 ; 1 \beta 84$ to $5 \beta 84$ is $3.42 \mathrm{~nm}$ with $\mathrm{K}=1.38$, and $1 \beta 84$ to $6 \alpha 84$ is 3.74 nm with $k=0.24$. This list includes only interactions with chromophores in the first 'monomer' unit; by symmetry there are identical interactions involving the chromophores in each of the other 'monomer' units of the $(\alpha \beta)_{3}$-trimer and the $(\alpha \beta)_{6}$-hexamer.

\section{Delocalization of excitation}

In Figs. 2-5 are shown the calculated decay profiles starting with equal excitation of all chromophores, for the $\beta$-subunit, the $\alpha \beta$-monomer, the $(\alpha \beta)_{3}$-trimer and the $(\alpha \beta)_{6}$-hexamer. The three

Table 2. Initial excitation transfer time constants $\left(\tau_{0}{ }^{1}=1 / \mathrm{k}_{0}{ }^{-1}\right.$, in ps) for energy relaxation from the chromophore initially excited in C-phycocyanin for different cluster sizes

\begin{tabular}{lcccc}
\hline & \multicolumn{4}{c}{ Chromophore initially excited } \\
& $\begin{array}{c}\text { No. of } \\
\text { chromophores }\end{array}$ & $\alpha 84$ & $\beta 84$ & $\beta 155$ \\
\hline$\beta$-Subunit & 2 & - & 230 & 59 \\
$\alpha \beta$-Monomer & 3 & 900 & 230 & 53 \\
$(\alpha \beta)_{3}$-Trimer & 9 & 29.1 & 11.8 & 29.7 \\
$(\alpha \beta)_{6}$-Hexamer & 18 & 7.4 & 7.7 & 6.6 \\
\hline
\end{tabular}

$k_{11}{ }^{\prime}$ is defined by Eq. 2 in the text. 


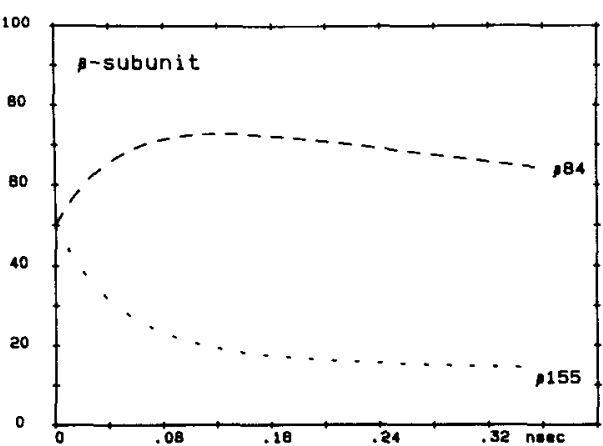

Figure 2. Calculation of excited state relaxation for the $\beta$-subunit of C-PC for the $\beta 84=f$ (fluorescer) chromophore (upper curve, dashed) and for the $\beta 155=\mathrm{s}$ (sensitizer) chromophore (lower curve, dotted). The calculation was carried out using the backward Euler method, using about 500 time steps that increase from 0.01 ps near $t=0$ to 50 ps at $t=5 \mathrm{~ns}$. The curves plotted are the sums (in percent of total excitation at time zero) of the results from two separate calculations with the initial excitation residing on each of the two chromophores, respectively.

types of chromophores in the monomer and higher aggregates are assumed to be populated equally initially. A few more realistic choices corresponding to excitations at 570,600 and $630 \mathrm{~nm}$ are shown in Fig. 6. Comparison of corresponding curves from Figs. 2-5 shows the effect of increasing cluster size on the initial relaxation process; this occurs significantly more rapidly in the hexamers and trimers than it does in the monomer or $\beta$-subunit. What is shown only in Figs. 5B,C but is quite clear from detailed inspection of the results of the calculations for the other cluster sizes, is that all of the chromophores of the same type (e.g., all of the $\beta 84$ chromophores in monomers 1 through 6 of the hexamer) come rapidly to essentially the same population level, regardless of which chromophore was initially excited. This occurs, for example, within the first 150 ps (to an identity of $\pm 1 \%$ in the

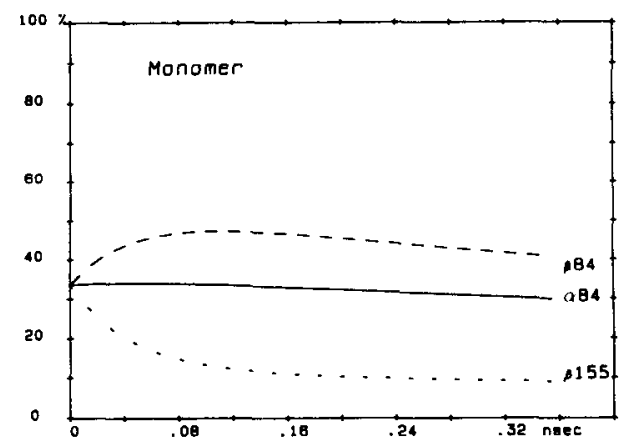

Figure 3. Calculated excited state relaxation for the $\alpha \beta$ monomer of C-PC for the $\alpha 84=\mathrm{m}$ (intermediate) chromophore (solid), the $\beta 84$ (dashed) and the $\beta 155$ chromophore (dotted). Calculation carried out as described in Fig. 2, except that each curve plotted is the sum of three components calculated for the initial excitation residing on each of the three chromophores, respectively.

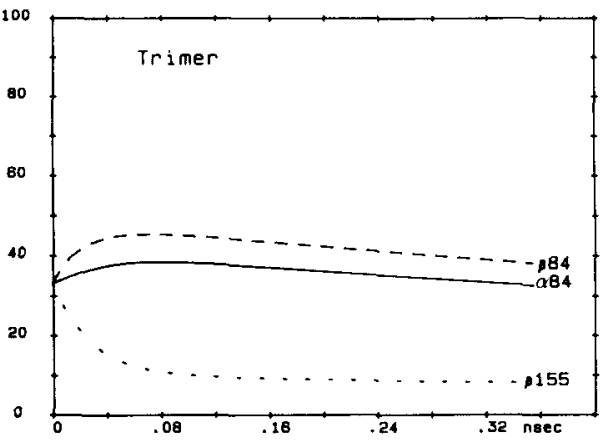

Figure 4. Calculated excited state relaxation for the $(\alpha \beta)_{3}$ trimer of C-PC for the $\alpha 84$ (solid), the $\beta 84$ (dashed) and the $\beta 155$ chromophore (dotted). Rate constants determined and calculations carried out as in Fig. 3, except that each curve plotted is the sum of the populations of all three chromophores of each class $(\alpha 84, \beta 84, \beta 155)$ as well as the sum of the results from three separate calculations with the initial excitation residing on one chromophore from each of the three different classes, respectively.

populations) for the run shown in Fig. 5A. From that time on the different populations are in a quasistationary state with respect to one another, apart from the relatively slow overall excited state decay. The relative populations during this later stage in the decay, presented in the top two lines of data in Table 3, are governed by 'uphill' and 'downhill' excitation transfer among the different chromophore classes, which are determined by the respective spectral overlap integrals. The fact that the sensitizer (s) chromophore population does not decay rapidly to zero is a reflection of the fact that 'uphill' energy transfer is significant for C-PC. This is implicit in the values for the overlap integrals presented in Table 1 , where the most favorable 'downhill' value from the $\beta 155$ chromophore to $\alpha$ is only 4 to 5 times more favorable than the most 'uphill' transfer from $\alpha 84$ or $\beta 84$ to $\beta 155$. If these analyses of the spectra are correct, then all of the excitation transfer steps of importance in C-PC must be considered to occur significantly in both directions.

Also presented in Table 3 is a summary of an analysis of the decay kinetics of the excited state populations in terms of a sum of two (Fig. 2) or three (Figs. 3-5) exponentials. Owing to the symmetry of these phycocyanin molecules, these are exactly the numbers of exponentials expected in each case from a direct solution of the differential equations (A. R. Holzwarth et al., 1987). For each species modeled, one of the exponentials has a decay constant that is simply $k_{\mathrm{F}}$, which was taken to be $0.67 \mathrm{~ns}^{-1}$ for our calculations. The other (one or) two decay constants are algebraic combinations of all of the transfer rate constants plus $k_{\mathrm{F}}$. Analytical expressions or numerical solutions for the values of these decay constants can be obtained directly from the matrix of coefficients of terms in the differ- 
A

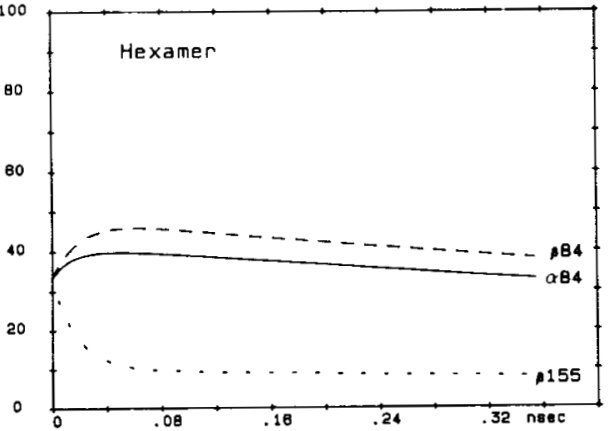

B

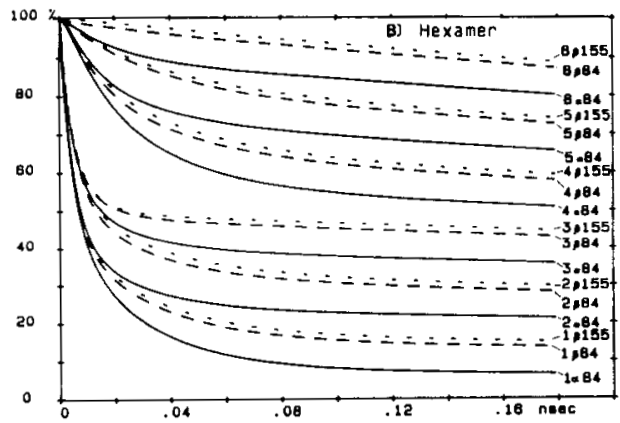

C

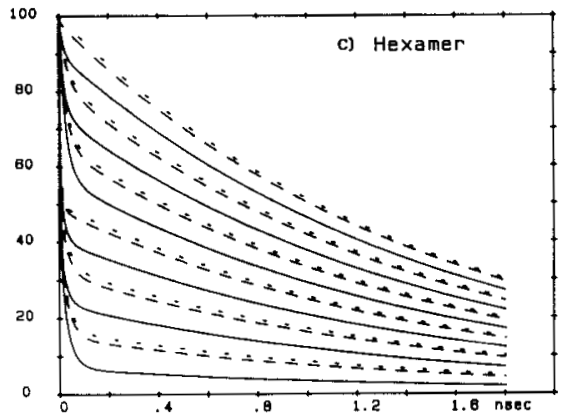

Figure 5. (A) Calculated excited state relaxation for the $(\alpha \beta)_{6}$-hexamer of C-PC for the $\alpha 84$ (solid), the $\beta 84$ (dashed) and the $\beta 155$ chromophores (dotted). Rate constants determined and calculation carried out as in Fig. 4, except that each curve plotted is the sum of the populations of all six chromophores. (B) Populations as a function of time for excited states of each of the 18 chromophores of the hexamer following initial excitation of the $1 \alpha 84$ chromophore. For clarity, a different plot style was chosen. The line coding is as in Fig. 3, starting with $1 \alpha 84$ (e.g. the $\alpha 84$ chromophore on the monomer unit 1) at the boltom and continuing with $1 \beta 84,1 \beta 155,2 \alpha 84$ through $6 \beta 155$. The fraction of excitation on each chromophore corresponds to the difference between two adjacent curves e.g. the fraction of excitation on $1 \alpha 84$ is the difference between the baseline and the solid line labeled $1 \alpha 84$, that for $1 \beta 84$ the difference between the line labeled such and the one labeled $1 \alpha 84$, etc. The difference between the base-line and topmost line $(6 \beta 155)$ corresponds to the total excited state population. (C) is as (B), but plotted out to $1.5 \mathrm{~ns}$.
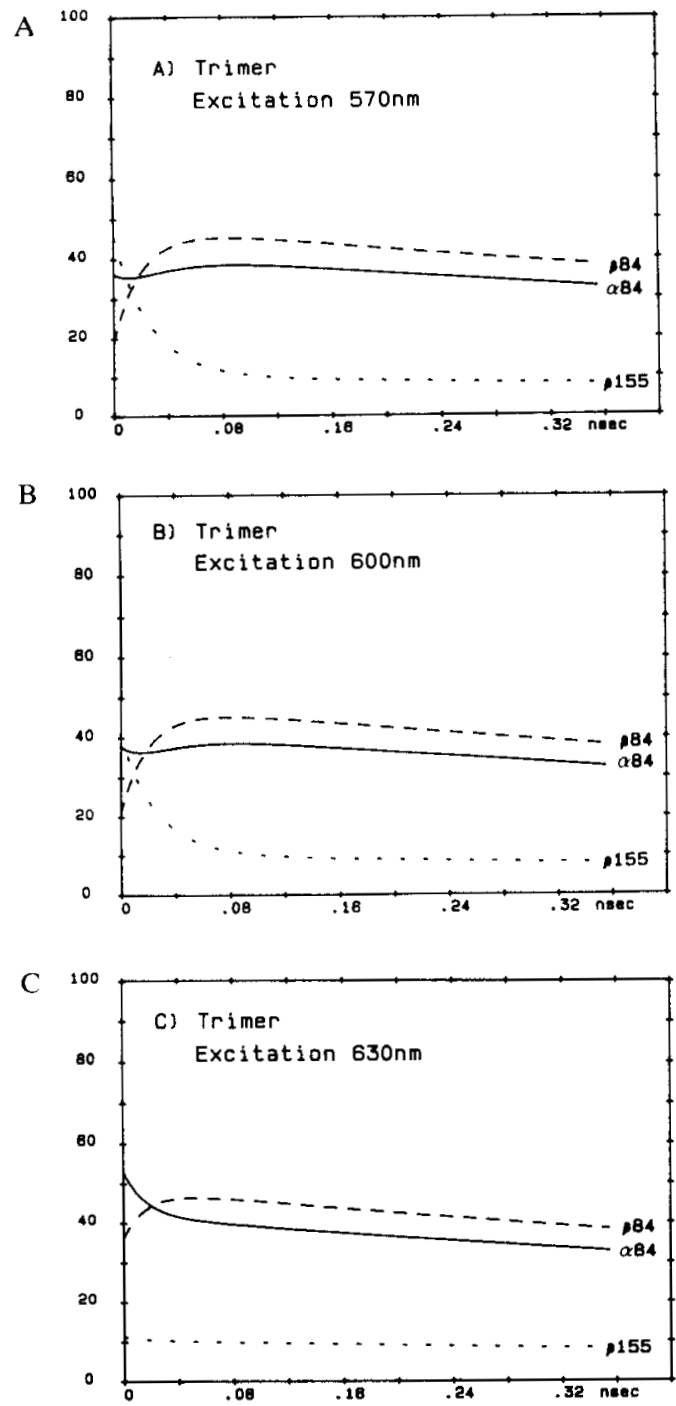

Figure 6. Calculated excited state relaxation for the $(\alpha \beta)_{3-}$ trimer of PC for the $\alpha 84$ (solid), the $\beta 84$ (dashed) and the $\beta 155$ chromophore (dotted). Rate constants determined and calculations carried out as in Fig. 4A, except that each curve plotted is the sum of the results obtained from three separate calculations with the initial excitation numbers for the three different chromophores determined from Fig. 1B. Simulations for excitation at 570 (A), 600 (B) and $630 \mathrm{~nm}(\mathrm{C})$.

ential equations, which gave identical solutions to the ones determined by the iterative procedures.

\section{DISCUSSION}

\section{General}

The significance of the results described in this manuscript is that, for the first time for a photosynthetic pigment protein, excitation transfer rates were calculated for a specified molecular structure determined by X-ray crystallography. It is appropri- 
Table 3. Kinetics of partitioning excitation among chromophore classes

\begin{tabular}{|c|c|c|c|c|}
\hline & $\begin{array}{c}\text { These } \\
\text { calculations }\end{array}$ & Synechococcus* $\dagger$ & M. laminosus $+\frac{\perp}{\mp}$ & M. laminosus \\
\hline$\beta$-Subunit & $\begin{array}{r}48 \\
1500\end{array}$ & & & $\begin{array}{r}191 \| \\
1041 \|\end{array}$ \\
\hline$(\alpha \beta)$-Monomer & $\begin{array}{r}45 \\
700 \\
1500\end{array}$ & $\begin{array}{r}47 \\
200 \\
675,1320\end{array}$ & & $\begin{array}{r}272 \\
2200\end{array}$ \\
\hline$(\alpha \beta)_{3}$-Trimer & $\begin{array}{r}16 \\
27 \\
1500\end{array}$ & $\begin{array}{r}20 \\
122 \\
600,1300\end{array}$ & $\begin{array}{r}36 \\
203 \\
807,1420\end{array}$ & $\begin{array}{c}45-61^{\S} \\
1130-1640^{*}\end{array}$ \\
\hline$(\alpha \beta)_{6}$-Hexamer & $\begin{array}{r}15 \\
18 \\
1500\end{array}$ & $\begin{array}{r}10 \\
40-50 \\
1800\end{array}$ & & \\
\hline
\end{tabular}

${ }^{*}$ Holzarth, 1985; †Holzwarth et al., 1987; $\ddagger$ Wendler et al., 1986; sSchneider et al., 1984a; |Hefferle et al., 1984a.

Calculated values listed are the time constants (in ps) for the fast $\left(\tau_{1}\right)$ and intermediate $\left(\tau_{2}\right)$ of a three exponential analysis, with $k_{\mathrm{F}}=0.67 \mathrm{~ns}^{-1}$ for the rate constant of radiative plus non-radiative decay corresponding to $\tau_{3}$.

ate then to compare the calculated behavior with experimental data available from measurements of the identical or similar molecules. Before this will be done, it may be useful to discuss critically the experimental data used in the calculations, as well as the limitations of comparisons with currently available experimental data.

The three different types of chromophores in the molecule have geometries that can be specified to different degrees of certainty (Schirmer et al., 1986): All four rings are well defined for $\beta 155$, only two rings $(A, B)$ are defined for $\beta 84$ and educated guesses have been made for rings $C$ and $D$, and the definition of $\alpha 84$ is intermediate. While the interchromophore distances will be fairly well restricted by these data (but enter Eq. 1 at sixth power), the $\kappa$-factors entering with the power of two may be influenced considerably by the geometrical uncertainties. Also, the published structure with the highest resolution has been determined for Agmenellum quadruplicatum rather than for Mastigocladus laminosus, from which the spectral data have been extracted. Although the chromophore geometries of $A$. quadruplicatum PC are similar to the ones from $M$. laminosus, better refinements of the structures have to be awaited for more precise calculations. One also has to be aware that the crystal structure may not be identical to that in solution. It is already suggested from the crystallographic analysis (Schirmer et al., 1985, 1986) and reversible photochemistry (Siebzehnübl, 1986) that in particular the chromophore $\beta 84$ may not be rigidly immobilized in the protein.

Even if the geometries were precise, the distances and orientations used in Eq. 1 relate to charges rather than to masses. Again, the effect on $\kappa$ is expected to be more pronounced than on $R_{\mathrm{DA}}$. Scharnagl and Schneider (unpublished) have recently calculated the dipole transition orientations by molecular orbital methods applied previously to bile pigments (Wagnière and Blauer, 1976; Schneider et al., 1985b). The variations are generally less than $25 \%$ with extrema of 2 , which would change the rate constants by a factor of 1.6 and 4 , respectively. Only in the few cases with a very small $k$ factor together with a sizeable overlap integral or short $R_{\mathrm{DA}}$ is this approximation expected to affect the results noticeably. One such case is the transfer between $1 \alpha 84$ and $2 \beta 84$.

The next experimental factor in Eq. 1 is the overlap integral. There are three problems involved in deriving the spectra of the individual chromophores. The first one is the curve deconvolution. Mimuro et al. (1986) arrived at spectra similar to those we have presented in Fig. 1 by an independent analysis. The main differences are a more blue-shifted absorption of the $\beta 155$ chromophore, and a higher oscillator strength for the $\beta 84$ chromophore absorption in their analysis. Both would affect the overlap integrals and in particular the equilibrium distribution of the excitation energy, but the differences have been estimated to be $\leqslant 30 \%$. A second problem was the chromophore assignment at the beginning of this work. The two models with reversed assignment for the $\beta 84$ and $\beta 155$ chromophores were investigated in parallel during the calculations and give somewhat different results, but the results of Mimuro et al. (1986) and Siebzehnrübl et al. (1987) now strongly support the 'mfs model' with $\beta 84$ as fluorescer and $\beta 155$ as sensitizer, and only these data are published.

The third problem is that the same chromophore spectra have been used for the monomer, trimer and hexamer. Whereas the absorption (and CD) spectra of the subunits add up to that of the monomer (Mimuro et al., 1986; John et al., 1985; Switalski 
and Sauer, 1985), red shifts on the order of $6 \mathrm{~nm}$ occur in $\mathrm{PC}$ from $M$. laminosus (Mimuro et al., 1986; John et al., 1985) upon trimerization, which have been neglected in the calculations. The crystallographic data have been collected for molecules that are trimeric (Mastigocladus laminosus) or hexameric (Agmenellum quadruplicatum) in their most stable forms. There are small but measurable wavelength shifts and band narrowing of the trimer and hexamer spectra of other biliproteins, even in the absence of linker peptides, relative to those of the monomer (Glazer et al., 1973; MacColl and Berns, 1981). The spectroscopic differences become more pronounced in the presence of linkers, which are known to produce considerable red-shifts (Lundell et al., 1981) and other spectral changes possibly including exciton interactions (Lehner and Scheer, 1983). The results are then limited to small aggregates without linkers.

Additional factors of uncertainty in $\mathrm{Eq} .1$ are the fluorescence lifetime and the quantum yield. Both enter only to the first power, and their influence should then be small as compared to the other ones. It should be noted, however, that the different chromophores probably have different rate constants for relaxation to the ground-state, whereas a single one $\left(0.67 \mathrm{~ns}^{-1}\right)$ has been assumed throughout these calculations. There is also experimental evidence which suggests that individual chromophores may be present in different environments or conformations exhibiting different lifetimes (Hefferele et al., 1985; Switalski and Sauer, 1984).

Besides these factors affecting the quality of the calculation, there are also problems in the comparison with experimental data. The experiments have been carried out with pigments from a variety of organisms. For most of these organisms, detailed studies on the individual chromophore spectra are not available, and there are indeed small but noticeable differences in the shape and position among the absorption bands of different PC. Although the two $\mathrm{PC}$ molecules investigated by $\mathrm{X}$-ray crystal lography have remarkably similar internal structures, one cannot be certain that this similarity will hold for PC from other organisms. The spectral resolution factors for absorption and emission would then be different. The experiments have also been carried out with a wide variety of techniques, exhibiting rather different time and spectral resolution, photon fluxes, etc. (see Table 3). Furthermore, in some cases, such as with lasers, the exciting light may have a large degree of spatial anisotropy (plane polarization). The extent to which this is present will certainly influence any measurements that sample anisotropic behavior, and knowledge of the specific experimental parameters is important for comparison with model calculations. An important problem is the extraction of the kinetic parameters from the experimental curves. This depends critically on the model used (e.g. nonexponential, multiexponential, analysis of individual traces or global fit), and on the signal-to-noise of the data (see Hefferle et al., 1984a; Holzwarth, 1986 for discussion).

Nevertheless, with all of these reservations, we feel that the experimental basis is so much better defined for PC than for any other photosynthetic antenna complexes, that some comparison with published experimental results is warranted and interesting. Further refinement of the crystallographic and experimental data have to be awaited for a more precise analysis (see note added in proof).

\section{Effect of different aggregation state}

Comparison with steady state data. Mimuro et al. (1986) have performed an important and imaginative analysis of a detailed set of spectroscopic measurements of the $(\alpha \beta)_{3}$-trimer, the $\alpha \beta$-monomers and the separated $\alpha$ - and $\beta$-subunits of PC from $M$. laminosus, which was used to deduce information about the path of excitation energy transfer. The authors made use of preliminary X-ray data on crystals of the $(\alpha \beta)_{3}$-trimers; further refinements of this structure (Schirmer et al., 1985) show that it is very similar to the two halves of the (more highly resolved) $(\alpha \beta)_{6}$-hexamer structure of PC from $A$. quadruplicatum (Schirmer et al., 1986). It appears, therefore, that both experimental results and calculations for the PC molecules from these two organisms should be closely comparable. Our more detailed analysis reaches conclusions which are identical to those of Mimuro et al. (1986) in some cases, but there are also important differences. Throughout the discussion of these data, the $\mathrm{mfs}$ model with $\beta 84$ as the fluorescing and $\beta 155$ as the sensitizing chromophore is assumed, as has been done by Mimuro et al.

On the basis of steady state fluorescence polarization measurements Mimuro et al. (1986), concluded that, for the $\alpha \beta$-monomer, the dominant energy transfer is from the $\beta 155$ to $\beta 84$. This is in agreement with our calculations, which show that rate to be nearly five-fold larger than any other. The next largest is the back transfer between the same two chromophores, and transfer from $\beta 155$ to $\alpha 84$ is almost a factor of two slower than that. We agree with their conclusion that excitation transfer in the monomer occurs mainly within subunits rather than between them and that the observed fluorescence emission should be a superposition of strong contributions from the $\alpha 84$ and the $\beta_{\mathrm{f}}$ (probably $\beta 84-)$ chromophores. Our calculated relaxation rates for excitation on the $\alpha$ chromophore is only about four times the measured fluorescence decay rate. The relaxation of the $\beta$-chromophores with one another is almost ten times faster than that. This supports the relative isolation of the excitation on the two subunits in the $\alpha \beta$-monomer.

For the $(\alpha \beta)_{3}$-trimer the proximity of the $\alpha$ chromophore of one monomer unit to the $\beta 84$ of 
the next adjacent monomer introduces an important new energy transfer path. Indeed, our calculation indicates that this new transfer path is about $20 \%$ faster than any other in the $(\alpha \beta)_{3}$-trimer. We disagree, however, with the conclusion of Mimuro et al. (1986) that the $\alpha 84$ to neighboring $\beta 84$ and $\beta 155$ to $\beta 84$ transfer within each monomeric unit are the dominant transfer steps. Our calculations indicate that two other processes have quite similar rates: the back transfer from $\beta 84$ to the neighboring $\alpha 84$ chromophore and the transfer from $\beta 84$ of one subunit to the $\beta 84$ of each of the other two subunits. These two processes have important consequences for the observed spectroscopic properties of the $(\alpha \beta)_{3}$-trimer. The back-transfer between $\beta 84$ and $\alpha 84$ means that excitation is distributed significantly over both of these chromophores, and, just as in the $\alpha \beta$-monomer but here for a different reason, the observed fluorescence should have significant contributions from both of these chromophore classes. This is in contrast to the conclusion of Mimuro et al. that in the $(\alpha \beta)_{3}$-trimer the fluorescence should come almost entirely from the $\beta 84$ chromophore. In this sense it is important to note that the spectroscopic properties deduced for the $\alpha 84$ chromophore, both by Mimuro et al. and by us, indicate that it should behave more like an $f$ (fluorescing) than an s (sensitizing) chromophore.

The efficient transfer from $\beta 84$ of one monomeric unit of the $(\alpha \beta)_{3}$-trimer to $\beta 84$ of the other two units has important consequences for the fluorescence depolarization. The intermonomer transfer between $\alpha 84$ and $\beta 84$, together with the rapid delocalization of excitation among the $\beta 84$ chromophores, leads to a depolarization mechanism in the $(\alpha \beta)_{3}$-trimer that is not present in the $\alpha \beta$-monomer. This latter exchange among the $\beta 84$ chromophores is the immediate cause of depolarization, because the chromophores are nearly perpendicular $\left(86^{\circ}\right)$ to one another. There will be additional contributions owing to subsequent back transfers to $\alpha 84$ chromophores. This strong depolarization is in agreement with the experimental observations of Mimuro et $a l$., but they were led to quite different conclusions.

It is interesting in this respect to compare the experimental steady state fluorescence polarization data, with the ones calculated for the three cluster sizes, $\beta$-subunit, monomer and trimer (Fig. 7). The

Figure 7.Calculated $(O)$ and experimental $\left(x: \lambda_{\mathrm{cm}}=670\right.$ $\left.\mathrm{nm}, 0: \lambda_{\mathrm{cm}}=656 \mathrm{~nm}\right)$ degree of fluorescence polarization spectra for the $\beta$-subunit (a), the monomer (b) and the trimer of PC (c). Experimental data from Mimuro et al. (1986) for $M$. laminosus $\left(\lambda_{\mathrm{cm}}=670 \mathrm{~nm}\right)$, and from Grabowski (private communication, 1986) for Phormidium luridum $\left(\lambda_{\mathrm{em}}=656 \mathrm{~nm}\right)$. An emission wavelength of $670 \mathrm{~nm}$ has been used throughout the calculations. The difference for emission at $655 \mathrm{~nm}$ (corresponding to the $P$. luridum monomer data), is smaller than the diameter of the circles (not shown).

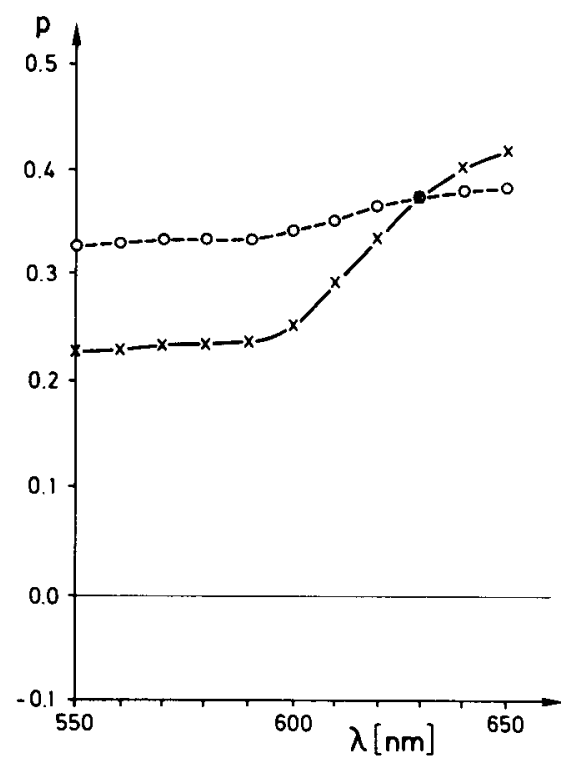

(a)

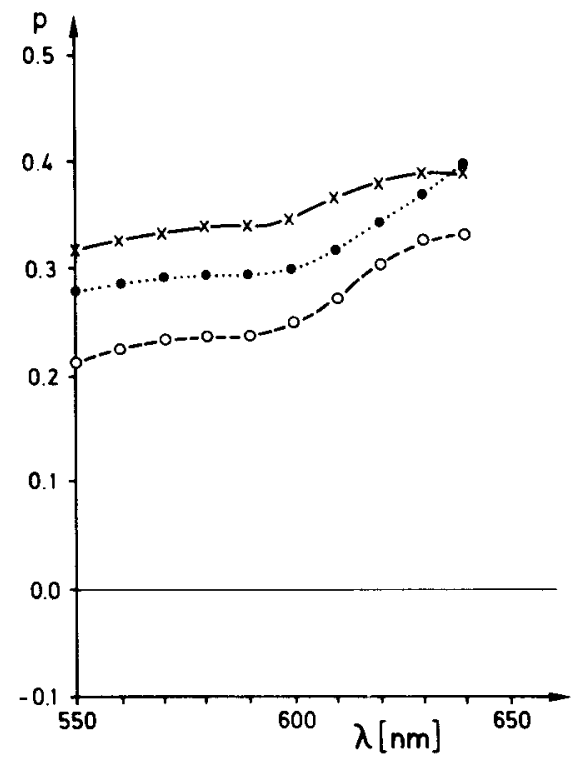

(b)

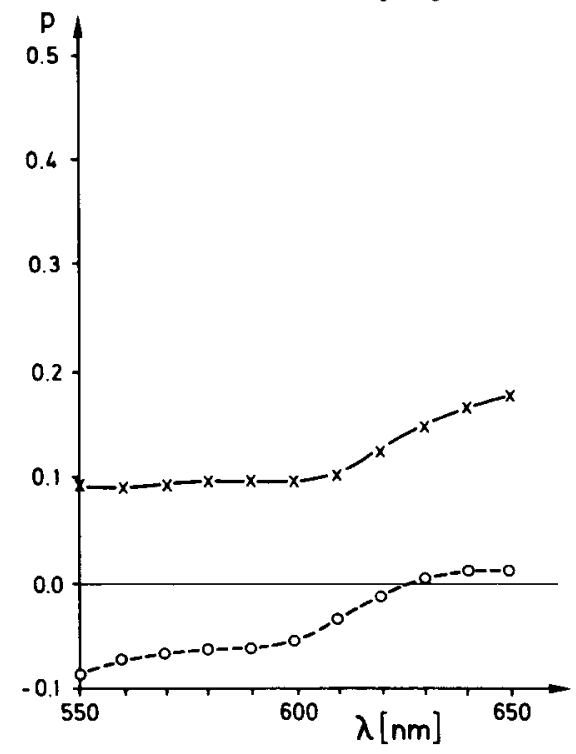


break-points in the fluorescence polarization spectra agree well with the experimental data, but the agreement of the absolute values is only moderate. In view of the importance of $\kappa$ for these calculations, the discrepancies may relate to the uncertainties discussed above for this factor. For the $\beta$ subunit, the calculated degree of polarization is too high throughout most of the spectral range. Lower experimental values can principally be reconciled from the fact that generally the maximum value for isotropic samples, 0.5 , is not attained, probably due to a non-parallel orientation of absorption and emission dipoles of each chromophore, and also to experimental limitations. However, this would not explain the small increase at longer wavelengths, which is much less pronounced than in the experimental data of Mimuro et al. (1986). For the monomer and trimer, the calculated values are even smaller than the experimental ones throughout the spectrum. It should be noted, that the difference among experiments of different PC monomers, e.g. the ones from $M$. laminosus (Mimuro et al., 1986) and Phormidium luridum (Grabowski, personal communication) is not negligible, but is of the same size as the difference between experimental and calculated values.

The $\beta 84$ to $\beta 84$ transfer is especially important in understanding an additional study carried out by Mimuro et al. (1986). They produced a 'treated' trimer, by reconstituting $\beta$-subunits with $\alpha$ '-subunits, where the $\alpha 84$ chromophore had been modified chemically to shift the excited states to higher energy (absorption to shorter wavelength, approx. $450 \mathrm{~nm}$ ). They found that the excitation polarization spectrum of the $\left(\alpha^{\prime} \beta\right)_{3}$-trimer was similar to that of the untreated $\alpha \beta$-monomer. The observed polarization values were distinctly higher than those of either the $\beta$-subunit or the unmodified $(\alpha \beta)_{3}$-trimer, and they reached the conclusion that energy transfer between adjacent monomeric units in $\left(\alpha^{\prime} \beta\right)_{3}$ does not occur. Our calculations show, however, that there should still be rapid excitation transfer among the three $\beta 84$ chromophores in the $\left(\alpha^{\prime} \beta\right)_{3}$-trimer. A principal difference will occur in that back transfer from $\beta 84$ to $\alpha 84$ should be missing.

In going from the $(\alpha \beta)_{3}$-trimers to the $(\alpha \beta)_{6^{-}}$ hexamers present in C-PC from $A$. quadruplicatum, three important new transfer steps become important across the boundary between the two trimeric parts of the $(\alpha \beta)_{6}$-hexamer. The transfer rates between $\beta 155$ chromophores (e.g. $1 \mathrm{~B} 2$ to $6 \mathrm{~B} 2,2 \mathrm{~B} 2$ to $4 \mathrm{~B} 2$, etc. in the notation of Schirmer et al. (1986) and corresponding to $1 \beta 155$ to $6 \beta 155$, etc. in our notation) and between $\alpha 84$ chromophores ( $1 \alpha 84$ to $4 \alpha 84,2 \alpha 84$ to $5 \alpha 84$, etc.) are more than three times faster than any other rates. The consequence is that excitation is very rapidly exchanged between the two trimeric units, even more rapidly than it flows from either of these chromophores to the $\beta 84$ (presumed fluorescer) chromophore. In the crystal, this excitation exchange occurs even more rapidly between the hexameric molecules because of the very rapid transfer between $\beta 84$ chromophores calculated to occur across the interface between adjacent hexameric molecules. All of these effects give a very strong component to excitation transfer along the direction of the threefold symmetry axis of the structure. Assuming that this is the same direction as the long axis of phycobilisome rods, it would provide for a very efficient path for excitation transfer to reach the allophycocyanin-containing core.

This picture of excitation transfer is somewhat different from one that has been popular in recent years. In that picture excitation transfer is considered to result in a rapid transfer from the $\alpha 84$ and $\beta 155$ chromophores in each trimeric unit to the $\beta 84$ chromophores in the same trimeric unit. The $\beta 84$ chromophores then constitute a kind of central cluster in channeling excitation along the rod axis and in the direction of the phycobilisome core. There is no doubt, based on our calculations, that these are important steps in the overall process. However, the efficient exchange of excitation between $\beta 84$ and $\alpha 84$ chromophores together with the efficient transfer between $\beta$ chromophores of adjacent trimeric units means that the $\alpha 84$ chromophores participate strongly' in increasing the crosssection of the excitation transfer channel along the rod axis. The depletion of excitation that arrived initially on a $\beta 155$ chromophore occurs significantly more slowly. Summing all transfers from and back transfers to $\beta 155$ from the other chromophore classes one calculates an expected lifetime of 17 ps for the decay of excitation from the collection of $\beta 155$ chromophores in the $(\alpha \beta)_{6}$-hexamer. This number will be essentially unchanged in the corresponding crystalline state. Even in the $\alpha \beta$-monomers or the $\beta$-subunit the corresponding time for energy loss from $\beta 155$ chromophores increases only to about $50 \mathrm{ps}$. The dependence of corresponding times for the $\alpha 84$ and $\beta 84$ chromophores shows a much stronger dependence on the degree of aggregation.

Comparison with kinetic data. In their pioneering fast kinetic studies of ground state bleaching and recovery of C-phycocyanin from Phormidium luridum, Kobayashi et al. (1979) found evidence for increasing excitation transfer rates with increasing degree of aggregation. This trend has been substantiated subsequently by other authors (Hefferle et al., 1984a,b; Holzwarth, 1985, Holzwarth et al., 1987; Switalski and Sauer, 1983; Wendler et al., 1986). This trend is in the same direction as that calculated. Quantitatively, the results from the different groups differ considerably, and the calculated rates are generally faster than the measured ones (Table 3). With the improvement of experimental techniques, however, there seems to be a trend to a more favorable comparison. The data will be compared in order of increasing aggregate size. 
The $\beta$-subunit showed two decay components for both the anisotropic and the isotropic relaxation in C-PC from $M$. laminosus (Hefferle et al., 1984a). For the anisotropic decay a component with a lifetime of only 0.19 ns was observed, along with a 1.04 ns lifetime, which is not far from the mean lifetime (1.23 ns) observed for the anisotropic decay of the $\alpha$-subunit. (This subunit also shows a biexponential decay, whose origin may relate to an inherent heterogeneity (Switalski and Sauer, 1983; Hefferle et al., 1984a) and/or aggregation (Glazer et al., 1973; John, 1986). Because each decay curve was analyzed in terms of only two exponentials, it is reasonable to assume that the 0.19 ns lifetime arises from excitation transfer between the two chromophores in a $\beta$-subunit and that the longer lifetime reflects a mean of intrinsic decays. The Förster calculation for the $\beta$-subunit (Fig. 2) shows two distinct phases in the decay. The slower one is that determined by the 1.5 ns lifetime assumed for the excited state lifetime. The faster phase, which results from excitation transfer between chromophores, is nearly complete by $0.20 \mathrm{~ns}$. Its $1 / \mathrm{e}$ time is $48 \mathrm{ps}$, which is appreciably shorter than the resolved short experimental component. Perhaps some, but probably not all, of this discrepancy is attributable to the inability of the data to be resolved meaningfully into three or more components. At the same time, the isolated $\beta$-subunits also probably exist as $\beta_{2}$-dimers in aqueous solution (Glazer et al., 1973; John, 1986). If these dimers are arranged in a non-symmetric fashion, then that will certainly results in increased complexity in the decay.

For the $\alpha \beta$-monomer, the $(\alpha \beta)_{3}$-trimer or the $(\alpha \beta)_{6}$-hexamer species there is no reason to question the currently accepted degrees of aggregation; however, the homogeneity of the preparations used in some studies has not been confirmed directly by the investigators involved. The investigation of Hefferle et al. (1984a) shows both the isotropic and anisotropic decays resolved into two components for both the $\alpha \beta$-monomers and $(\alpha \beta)_{3}$-trimer. In $M$. laminosus $\mathrm{PC}$ the $(\alpha \beta)_{6}$-hexamer is not stable in aqueous solution. For the $\alpha \beta$-monomer the short lifetime of $0.25 \mathrm{~ns}$ for the anisotropic decay is even longer than the corresponding value for the $\beta$-subunit. This could reflect a contribution (unresolved) from the slow excitation transfer from the $\alpha 84$ chromohore predicted by the calculations. Because the values of $\kappa^{2} / R^{6}$ for the closest interactions ( $\beta 84$ and $\beta 155$ chromophores) are about the same for the $\beta$-subunit and for the $\alpha \beta$-monomer, there is essentially no difference in the $45-48$ ps lifetimes calculated for the initial excitation relaxation. These calculated values are significantly shorter than those resolved by Hefferle et al. (1984), however.

In the case of the $\alpha \beta$-monomers there is only one chromophore of each type present, so there is no conceptual problem in comparing results of the calculation with the fast relaxation of the anisotropic fluorescence, apart from the fact that no wavelength resolution was attempted by Hefferle et al. In the case of the $(\alpha \beta)_{3}$-trimer, however, there are three chromophores of each class per molecule. Thus, polarization anisotropy will be lost not only because of transfer from one chromophore class to another but also because of transfer among the chromophores within each class. This occurs also in the $(\alpha \beta)_{6}$-hexamers and higher aggregates, of course. In principle, because we know the chromophore orientations and separations, it is possible to model the relaxation observed under any particular set of experimental conditions. The wavelengths of excitation and emission need to be considered in this modeling, however, and these are not specified sufficiently in the experiments of Hefferle et al. (1984a).

The most rapid relaxation of the anisotropic fluorescence for the $(\alpha \beta)_{3}$-trimer of C-PC from $M$. laminosus occurs in 62 ps. Not only is this appreciably faster than the corresponding components from the $\beta$-subunit or the $\alpha \beta$-monomer, but it is in the range of values expected on the basis of the calculations (16 and $27 \mathrm{ps,} \mathrm{Table} \mathrm{3).} \mathrm{Perhaps} \mathrm{a} \mathrm{more}$ meaningful comparison for this initial anisotropy relaxation is with the rates of relaxation of the populations of the initially excited chromophores at time zero (Table 2). The calculated range is between 6 and $100 \mathrm{ps}$ in the $(\alpha \beta)_{3}$-trimer depending on which chromophores are excited and monitored by the fluorescence measurement. It is significant to note that these short lifetimes are seen experimentally only in the anisotropic fluorescence decay; the initial stages of the measured relaxation of the isotropic fluorescence appears to be significantly longer for each cluster size that was studied.

Several attempts have been made to improve the wavelength resolution for the excitation and emission. In a study of the $(\alpha \beta)_{3}$-trimer C-PC from $M$. laminosus by Schneider et al. (1985a) three pairs of monochromatic ( $\pm 8 \mathrm{~nm}$ ) excitation and emission wavelengths were used with the repetitive streak camera technique. For these measurements, where each decay was resolved into only two components, a fast relaxation in the range from 35 to $61 \mathrm{ps}$ was found in both the isotropic and the anisotropic fluorescence decays, which became shorter with an increasing spectral separation between the excitation $(575$ or $600 \mathrm{~nm}$ ) and emission wavelengths $(590,620$ or $630 \mathrm{~nm})$. The relative amplitudes depend significantly on the wavelength of excitation and emission monitoring, which can in principle be compared with model calculations. In each case a slower decay in the range of 0.8 to $1.64 \mathrm{~ns}$ was observed as well.

In a related study, Schneider et al. (1985b) examined a suspension of microcrystals of the $(\alpha \beta)_{3^{-}}$ trimer of C-PC from $M$. laminosus. Because of signal-to-noise limitations, only the isotropic fluorescence was monitored, using $580 \mathrm{~nm}$ excitation 
and monitoring fluorescence at all wavelengths longer than $620 \mathrm{~nm}$. Here a 59 ps short relaxation is also observed; however, the second component of $0.49 \mathrm{~ns}$ lifetime is appreciably shorter than the long lifetime of $1.31 \mathrm{~ns}$ resolved from an $(\alpha \beta)_{3^{-}}$ trimer solution measurement made using the same wavelengths of excitation and monitoring. This difference was attributed to the occurrence in the $(\alpha \beta)_{3}$-trimer crystals of a fast relaxation from strongly coupled $\alpha 84$ and $\beta 84$ chromophores. No such consequence is expected based on the model that we have used for our calculations, however. The overall excited state relaxation should be limited ultimately by the 1.5 ns fluorescence lifetime in each case. Recently, a more detailed wavelengthresolved study of C-PC from Synechococcus 6301 (formerly called Anacystis nidulans) has been carried out by Holzwarth et al. (1987). Investigations of the $\alpha \beta$-monomer, the $(\alpha \beta)_{3}$-trimer and the $(\alpha \beta)_{6}$ hexamer in aqueous solution were done using a synchronously pumped mode-locked dye laser and single photon-timing detection. Analysis of the data was carried out using a global data analysis method that allows the extraction of all spectroscopically distinguishable components (eigenvectors) from a simultaneous deconvolution of data collected for the same sample using different excitation and emission wavelengths. In each case investigated, four such eigenvectors were extracted from the analysis (Table 3). In addition to the different lifetimes of excitation decay, one or two of the lifetimes were associated with the delayed formation of excited state population of the longer wavelength absorbing chromophore classes. Only the isotropic fluorescence decay was examined in this study.

Of the four eigenvectors seen for the $\alpha \beta$-monomers, the two shorter ones are attributable to excitation transfer from the $\mathrm{s}(47 \mathrm{ps})$ and from the $\mathrm{m}$ (200 ps) chromophores. The shorter of these is in excellent agreement with the dominant decay of the $s$ chromophore calculated. Furthermore, the data shown in Table 2 make it clear that the loss of excitation from the $\mathrm{mM}(\alpha 84)$ chromophore of the $\alpha \beta$-monomer should be almost 10 -fold slower than that from s chromophores for each model. Thus, there appears to be very good agreement between the Förster transfer calculations and the experimental results of Holzwarth for the $\alpha \beta$-monomers. (One must remember, of course, that the X-ray crystallographic measurements have not yet been reported for PC from Synechoccus 6301). The two longer decay components, 0.675 and 1.32 ns, show similar spectral shapes and no indications of rising (negative amplitude) phases at any wavelength. For the present we assume that these both arise from the $f$ chromophores and have the same unknown origin as the corresponding components reported previously for the $\alpha$-subunit, the $\beta$-subunit and $\alpha \beta$ monomers of PC molecules from other organisms (Hefferle et al., 1984a,b; Switalski and Sauer, 1984).
For the $(\boldsymbol{\alpha} \beta)_{3}$-trimer the four experimentally resolved components include a short wavelength s-type spectrum with a very fast $(20 \mathrm{ps})$ decay at short wavelength and a $35 \mathrm{ps}$ rising phase at longer wavelength. A second component resembling the $\mathrm{m}(\alpha 84)$ chromophore is associated with a 122 ps lifetime. Similar properties have been observed using the global analysis approach applied to the $(\alpha \beta)_{3}$ trimer of PC isolated from $M$. laminosus (Wendler et al., 1986). Again, these values are in general agreement with values derived from the calculational models, as summarized in Table 3 . The properties of the $(\alpha \beta)_{6}$-hexamer from Synechococcus (Holzwarth, 1985; Holzwarth et al., 1987) are similar to those of the $(\alpha \beta)_{3}$-trimer, except that the intermediate decay component of $0.55 \mathrm{~ns}$ has only a very small amplitude. The decay can be described almost as well using only three components, with a somewhat longer lifetime, $1.8 \mathrm{~ns}$, associated with the $\mathrm{f}$ chromophore, and still shorter lifetimes of $10 \mathrm{ps}$ and $40-50 \mathrm{ps}$ associated with the $\mathrm{s}$ and $\mathrm{m}$ chromophores, respectively. The latter numbers are of the same magnitude as those predicted by the models, and the experimental data are in general agreement with the prediction that excitation transfer should be more rapid for the larger cluster sizes.

It should be possible to make a more stringent comparison between data of the type collected and analyzed by Holzwarth and the calculations. This will require a more detailed specification of the fraction of each chromophore class excited at each excitation wavelength and the fraction of each class of emission detected at each fluorescence wavelength. It should then be possible to simulate the individual response profiles under each set of wavelength conditions. Not only will this be helpful in verifying the general validity of the Förster transfer model applied to PC, but it should also help to confirm the assignment of the $\beta$-chromophores as having $s$ or $f$ function. Investigations along these lines are currently under way.

\section{CONCLUSION}

Excitation energy transfer in C-phycocyanin calculated using the Förster inductive resonance mechanism appears to be compatible with experimental measurements of isotropic fluorescence decay and of depolarization kinetics. In particular this mechanism can account for the fastest energy transfer times hitherto determined experimentally for this system. Based on these observations and the relatively long interchromophore distances determined from X-ray crystallographic analysis, we conclude that Förster transfer is sufficient to account for excitation transfer in C-phycocyanin. Although there is qualitative agreement between the calculated rates and experimentally measured quantities, further refinements in both the structural data and the spectroscopic 
measurements are needed before a quantitative comparison is meaningful. Nevertheless, this first detailed excitation transfer model calculation for a photosynthetic pigment protein is encouraging that much useful information will be obtained as additional kinetic and structural data become available in the near future.

Acknowledgements - Part of the spectra used for the deconvolution are unpublished results provided by $\mathrm{R}$. Fischer in the laboratory of H.S. We are indebted to T. Schirmer (MPI für Biochemic, Martinsried) and A. R. Holzwarth (MPI für Strahlenchemie, Mülheim) for providing unpublished data and many helpful discussions. We thank E. Jörn and P. Schuster (Mathematisches Institut der Universität, München) for help in choosing the numeric methods for solving the differential equations. The help of C. Bubenzer and S. Schoy in preparing the manuscript is acknowledged. This work was supported by the Deutsche Forschungsgemeinschaft (SFB143 and the CIP botany computer facilities, H.S.), and by the Alexander-von-Humboldt Stiftung, Bonn (award to K.S.), and by the Director, Office of Energy Research, Office of Basic Energy Sciences, Division of Biological Energy Conversion of the US Department of Energy under Contract DE-AC03-76SFO0098.

\section{REFERENCES}

Cogdell, R. J. and H. Scheer (1985) Circular dichroism of light-harvesting complexes from purple photosynthetic bacteria. Photochem. Photobiol. 42, 669-678.

Dale, R. E. and J. Eisinger (1975) Polarized excitation energy transfer. In Biochemical Fluorescence Concepts (Edited by R. F. Chen and H. Edelhoch), Vol. 1, pp. 115-284. Marcel Dekker, New York.

Förster, T. (1948) Zwischenmolekulare Energiewanderung und Fluoreszenz. Ann. Physik 2, 55-75.

Förster, T. (1967) Mechanisms of energy transfer. In Comprehensive Biochemistry (Edited by $\mathbf{M}$. Florkin and E. H. Stotz), Vol. 22, pp. 61-80. Elsevier, Amsterdam.

Glazer, A. N., S. Fang and D. M. Brown (1973) Spectroscopic properties of C-phycocyanin and of its $\alpha$ and $\beta$ subunits. J. Biol. Chem. 248, $5679-5685$

Grabowski, J. and E. Gantt (1978a) Photophysical properties of phycobiliproteins from phycobilisomes: Fluorescence lifetimes, quantum yields, and polarization spectra. Photochem. Photobiol. 28, 39-45.

Grabowski, J. and E. Gantt (1978b) Excitation energy migration in phycobilisomes: Comparison of experimental results and theoretical predictions. Photochem. Photobiol. 28, 47-54.

Hefferle, P., P. Geiselhart, T. Mindl, S. Schneider, W John and H. Scheer (1984a) Time-resolved polarized fluorescence of C-phycocyanin and its subunits from Mastigocladus laminosus. Z. Naturforsch. 39c, 606-616.

Hefferle, P., W. John, H. Scheer and S. Schneider (1984b) Thermal denaturation of monomeric and trimeric phycocyanins studied by static and polarized timeresolved fluorescence spectroscopy. Photochem. Photobiol. 39, 221-232.

Holzwarth, A. R. (1985) Energy transfer in phycobilisomes. In Antennas and Reaction Centers of Photosynthetic Bacteria (Edited by M. E. Michel-Beyerle), pp. 45-52. Springer-Verlag, Berlin.

Holzwarth, A. R., J. Wendler and G. W. Suter (1987) Picosecond energy transfer kinetics and time-resolved fluorescence spectra of C-phycocyanin from syne. chococcus 6301 as a function of the aggregation state. Biophys. J. 51, 1-12.
John, W. (1986) Phycocyanine aus Mastigocladus laminosus und Spirulina platensis: Biochemische Charakterisierung, Spektroskopie und Energietransfer in Abhängigkeit vom Aggregationszustand, Ph.D. thesis, Universität, München.

John, W., R. Fischer, S. Siebzehnrübl and H. Scheer (1985) C-phycocyanin from Mastigocladus laminosus: Isolation and properties of subunits and small aggregates. In Antennas and Reaction Centers of Photosynthetic Bacteria (Edited by M. E. MichelBeyerle), pp. 17-25. Springer-Verlag, Berlin.

Kobayashi, T., E. O. Degenkolb, R. Bersohn, P. M. Rentzepis, R. MacColl and D. S. Berns (1979) Energy transfer among the chromophores in phycocyanins measured by picosecond kinetics. Biochemistry 18, 5073-5078.

Lehner, H. and H. Scheer (1983) Circular dichroism of C-phycocyanin: Origin of optical activity in denatured biliproteins and evidence for an intermediate during unfolding. Z. Naturforsch. 38c, 353-358.

Lundell, D. J., R. C. Williams and A. N. Glazer (1981) Molecular architecture of a light-harvesting antenna-In vitro assembly of the rod substructures of Synechococcus-6301 phycobilisomes. J. Biol. Chem. 256, 3580-3592.

MacColl, R. and D. S. Berns (1981) Biliproteins: Some relations among aggregation states, spectra and excitation energy transfer. Isr. J. Chem. 21, 296-300.

Mimuro, M., P. Füglistaller, R. Rümbeli and H. Zuber (1986) Functional assignment of chromophores and energy transfer in C-phycocyanin isolated from the thermophilic cyanobacterium Mastigocladus laminosus. Biochim. Biophys. Acta 848, 155-166.

Sauer, K. (1975) Primary events in the trapping of energy. In Bioenergetics of Photosynthesis (Edited by Govindjee), pp. 115-181. Academic Press, New York.

Sauer, K. (1986) Photosynthetic light reactionsphysical aspects. In Encyclopedia of Plant Physiology, Vol. 19 Photosynthetic Membranes (Edited by L. A. Staehelin and C. J. Arntzen), Ch. 2. Springer-Verlag, Berlin.

Scheer, H. (1981) Biliproteine Angew Chem. 93, 230-250; Angew. Chem. Int. Ed. 20, 241-261.

Scheer, H. (1982) Phycobiliproteins: molecular aspect of photosynthetic antenna system. Light Reaction Path of Photosynthesis (Edited by F. K. Fong), pp. 7-45. Springer-Verlag, Berlin.

Schirmer, T., W. Bode, R. Huber, W. Sidler and H. Zuber (1985) X-ray crystallographic structure of the light-harvesting biliprotein C-phycocyanin from the thermophilic cyanobacterium Mastigocladus laminosus and its resemblance to globin structures. J. $\mathrm{Mol}$. Biol. 184, 257-277.

Schirmer, T., R. Huber, M. Schneider, W. Bode. M. Miller and M. L. Hackert (1986) Crystal structure analysis and refinement of $2.5 \mathrm{~A}$ of hexameric C-phycocyanin from the cyanobacterium Agmenellum quadruplicatum. The molecular model and its implications for light harvesting. J. Mol. Biol. 188, 651-676.

Schneider, S., P. Geiselhart, T. Mindl, F. Dörr, W. John, R. Fischer and H. Scheer (1985a) Picosecond timeresolved polarized fluorescence decay of phycobilisomes and constituent biliproteins isolated from Mastigocladus laminosus. In Antennas and Reaction Centers of Photosynthetic Bacteria (Edited by M. E. Michel-Beyerle), pp. 26-35. Springer-Verlag. Berlin.

Schneider, S.. P. Geiselhart, C. Scharnagl, T. Schirmer, W. Bode, W. Sidler and H. Zuber (1985b) Fluorescence behaviour of crystallized C-phycocyanin (trimer) from Mastigocladus laminosus. In Antennas and Reaction Centers of Photosynthetic Bacteria (Edited by M. E. Michel-Beyerle), pp. 36-44. Springer-Verlag, Berlin. Shampine, L. F. and M. K. Gordon (1975) Computer Solution of Ordinary Differential Equations. W. H. 
Freeman, San Francisco.

Siebzehnrübl, S. (1986) Untersuchungen zur Photochemie von partiell denaturiertem Phycocyanin aus Mastigocladus laminosus und dessen Untereinheiten, Diploma thesis, Universität München.

Siebzehnrübl, S., R. Fischer and H. Scheer (1987) Chromophore assignment in phycocyanin from Mastigocladus laminosus. Z. Naturforsch. C. 42, 258-262.

Switalski, S. C. (1986) Picosecond spectroscopic studies of energy transfer in phycobiliproteins and model dye systems. Ph.D. Thesis, University of California, Berkeley.

Switalski. S. C. and K. Sauer (1984) Energy transfer among the chromophores of C-phycocyanin from
Anabaena variabilis using steady-state and time-resolved fluorescence spectroscopy. Photochem. Photobiol. 40, 423-427.

Teale, F. W. J. and R. E. Dale (1970) Isolation and spectral characterization of phycobiliproteins. Biochem. J. 116, 161-169.

Wagniere, G. and G. Blauer (1976) Calculations of optical properties of biliverdin in various conformations. J. Am. Chem. Soc. 98, 7806-7810.

Wendler, J., W. John, H. Scheer and A. R. Holzwarth (1986) Energy transfer in trimeric C-phycocyanin studied by picosecond fluorescence kinetics. Photochem. Photobiol. 44, 79-85.

\section{Note Added in Proof}

The assignment of chromophores beta- 155 as " $\mathrm{s}$ " and beta- 85 as " $\mathrm{f}$ " has recently been confirmed independently from linear dichroism measurement on PC crystals (Schirmer et al., submitted for publication). We are aware of the recent resolution improvement on the crystal structures of PC from.M. laminosus and A. quadruplicatum (Schirmer et al., submitted for publication). Calculations with these data are in progress. We thank the authors for communication of unpublished results. 